\title{
Structured postoperative physiotherapy in patients with cervical radiculopathy: 6-month outcomes of a randomized clinical trial
}

\author{
Johanna Wibault, PhD, PT, ${ }^{1}$ Birgitta Öberg, PhD, PT, ${ }^{1}$ Åsa Dedering, PhD, PT, ${ }^{2,3}$ \\ Håkan Löfgren, PhD, MD, ${ }^{4}$ Peter Zsigmond, PhD, MD, ${ }^{5}$ and Anneli Peolsson, $\mathrm{PhD}, \mathrm{PT}{ }^{1}$ \\ 'Division of Physiotherapy, Department of Medical and Health Sciences, Linköping University; ${ }^{5}$ Department of Neurosurgery, \\ Linköping University Hospital, Linköping; '2Division of Physiotherapy, Department of Neurobiology, Care Sciences and Society, \\ Karolinska Institutet; ${ }^{3}$ Function Area Occupational Therapy and Physiotherapy, Allied Health Professionals Function, Karolinska \\ University Hospital, Stockholm; and ${ }^{4}$ Neuro-Orthopedic Center, Ryhov Hospital, Jönköping, Sweden
}

OBJECTIVE Structured physiotherapy has been suggested as treatment before as well as after surgery to improve clinical outcomes in patients with cervical radiculopathy (CR), but randomized clinical trials to inform evidence-based clinical guidelines for the treatment of patients with $\mathrm{CR}$ after surgery are lacking. The aim of this study was to compare the results of structured postoperative physiotherapy combining neck-specific exercises with a behavioral approach to a standard postoperative approach in patients who had undergone surgery for cervical disc disease with CR at 6 months after surgery.

METHODS Patients with cervical disc disease and persistent $C R$ who were scheduled for surgery were randomized preoperatively to structured postoperative physiotherapy $(n=101)$ or a standard postoperative approach $(n=100)$. The latter included pragmatic physiotherapy in accordance with the usual Swedish postoperative care. Outcome measures included patient-reported neck disability as measured with the Neck Disability Index (NDI), intensity and frequency of neck and arm pain, global outcome of treatment, and expectation fulfillment, as well as enablement.

RESULTS Patients who received structured postoperative physiotherapy reported greater expectation fulfillment ( $p$ $=0.01)$, and those who attended at least $50 \%$ of the treatment sessions reported less neck pain frequency $(p=0.05)$, greater expectation fulfillment $(p=0.001)$, and greater enablement $(p=0.04)$ compared with patients who received the standard postoperative approach. No other difference between treatment groups was found ( $p>0.15)$. The NDI and neck and arm pain intensity were improved in both groups at 6 months after surgery $(p<0.001)$. Additional use of postoperative physiotherapy was reported by $61 \%$ of the patients who received the standard postoperative approach.

CONCLUSIONS The results from this first randomized clinical trial of postoperative physiotherapy showed only minor additional benefit of structured postoperative physiotherapy compared with standard postoperative approach 6 months postoperatively in patients who underwent surgery for cervical disc disease with CR. Patients who received structured postoperative physiotherapy reported higher expectation fulfillment, and many patients in the standard postoperative approach group perceived a need for additional treatments after surgery, suggesting that patients with CR are in need of further postoperative support. The results confirm that neck-specific exercises are tolerated postoperatively by patients with $\mathrm{CR}$, but more studies of postoperative physiotherapy are needed to inform clinical guidelines for this patient group.

Clinical trial registration no.: NCT01547611 (clinicaltrials.gov)

https://thejns.org/doi/abs/10.3171/2017.5.SPINE16736

KEY WORDS cervical radiculopathy; postoperative physiotherapy; neck exercises; Neck Disability Index; pain outcomes; enablement

ABBREVIATIONS ACDF = anterior cervical decompression and fusion; $\mathrm{CR}=$ cervical radiculopathy; $\mathrm{NDI}=$ Neck Disability Index; $\mathrm{PCF}=$ posterior cervical foraminotomy; $\mathrm{PEI}=$ Patient Enablement Instrument; RCT = randomized clinical trial; $\mathrm{SA}$ = standard postoperative approach; $\mathrm{SPT}$ = structured postoperative physiotherapy; VAS = visual analog scale.

SUBMITTED June 22, 2016. ACCEPTED May 4, 2017.

INCLUDE WHEN CITING Published online November 3, 2017; DOI: 10.3171/2017.5.SPINE16736. 


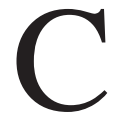
ERVICAL radiculopathy (CR) caused by disc disease is a painful and disabling condition characterized by radicular arm pain in combination with neurological impairments in 1 or more affected nerve-root distributions. ${ }^{3}$ Additionally, neck pain is common, ${ }^{4}$ and the symptoms are often accompanied by physical and psychological disability, absence from work because of health issues, and reduced health and quality of life. ${ }^{6}$

The prognosis of CR is considered to be favorable over time, but patients who report no improvement in symptoms may be referred to surgery. ${ }^{1}$ Overall, fair to very good results have been reported with respect to arm pain and neurological symptoms after surgery depending on the outcome measure used and duration of follow-up. ${ }^{19,32}$ The effects on neck function are less clear, ${ }^{13,27}$ and more extensive and structured physiotherapy has been suggested as necessary to improve clinical outcomes regarding persistent activity limitations and participation restriction as well as impairments in neck-related physical function in CR patients after surgery. ${ }^{13,14,27}$ Structured physiotherapy combining neck-specific exercises with a behavioral approach may be suggested as treatment for patients with CR before as well as after surgery.,26 How well patients with $\mathrm{CR}$ can tolerate a more active approach is unknown, and loading of the cervical spine in exercises had been questioned by clinicians. The proportion of CR patients who seek additional physiotherapy after surgery is unknown as well. Randomized clinical trials (RCTs) of postoperative physiotherapy to inform evidence-based clinical guidelines for the treatment of patients who have undergone surgery for cervical disc disease with CR are lacking. ${ }^{1}$

The aim of the present prospective RCT was to compare structured postoperative physiotherapy combining neck-specific exercises with a behavioral approach to a standard postoperative approach in patients who underwent surgery for cervical disc disease with $\mathrm{CR}$.

\section{Methods \\ Design}

This study was a multicenter parallel group RCT (January 2010-December 2014) with a 1:1 allocation ratio and a blinded assessor to collect the outcome measures. The study was approved by the regional ethical review board, performed according to the Declaration of Helsinki, and registered with clinicaltrials.gov (registration no.: NCT01547611), and the study protocol was published. ${ }^{25}$ The study was reported according to the Consolidated Standards for Reporting of Trials (CONSORT) guidelines. ${ }^{22}$

\section{Participants}

Patients who had persistent CR symptoms and clinical findings compatible with disc disease verified on MRI and who were scheduled for surgery were recruited from 4 spinal centers in Sweden. The eligibility criteria for the study are described in Table 1. Patients were given oral and written information about the study. A total of 202 patients (mean age 50 years, SD 8.4 years; 105 men and 97 women) with a median duration of symptoms of 12 months (range 2-288 months) were included after providing informed consent. The patients were randomized preoperatively
TABLE 1. Eligibility criteria for participation in the study

\begin{tabular}{cc}
\hline \multicolumn{1}{c}{ Inclusion Criteria } & \multicolumn{1}{c}{ Exclusion Criteria } \\
\hline Age 18-70 yrs & Previous cervical surgery \\
Presenting w/ persistent & Fracture or traumatic subluxation of \\
symptoms of CR of at & cervical column \\
least 2 mos' duration & Myelopathy \\
Clinical findings compatible & Malignancy or spinal tumor \\
w/ MRI-verified cervical & Spinal infection \\
disc disease & Any systemic disease implying a contra- \\
Referred for surgery & indication to rehabilitation \\
btwn February 2009 \& & Fibromyalgia or generalized myofascial \\
November 2012 at one & pain, persistent or recurrent severe \\
of 4 spinal centers in the & back pain \\
south of Sweden & Diagnosed mental disorder \\
& Known drug or alcohol addiction \\
& Not being fluent in the Swedish language \\
\hline
\end{tabular}

by the central project leader to receive either structured postoperative physiotherapy (SPT group) or a standard postoperative approach (SA group) (Fig. 1). The central project leader was not involved in any of the treatments or measurements but was in contact with the physiotherapists working with the SPT group. Simple randomization was performed using a random computer list developed by an independent statistician.

\section{Surgery}

The surgical procedures included anterior cervical discectomy and fusion $(\mathrm{ACDF})(\mathrm{n}=163)$ or posterior cervical foraminotomy $(\mathrm{PCF})$ with or without laminectomy $(\mathrm{n}=38)$ in accordance with current clinical practice at the 4 spinal centers. Cages filled with bone substitute or autologous bone collected during decompression were used to fuse the segment after the disc and osteophyte(s) were removed in 1-level ACDF $(n=99)$. In 2-level $(n=62)$ and 3-level $(n=2)$ fusions, an anterior plate was added in most cases to achieve primary stability. Each spinal center used cages that were standard for that center. No iliac crest graft was taken. PCF was performed at 1 level $(n=7), 2$ levels $(n=15)$, or 3 or more levels $(n=16)$ without fusion. One patient was excluded from the study because of a cancelled surgery (Fig. 1).

\section{Standard Initial Postoperative Care at the Spinal Centers}

During the first 6 weeks after surgery, the same initial postoperative regimen was applied in all cases (Fig. 1). The patients were given advice about good posture and ergonomics, information about movements and tasks to avoid during the first postoperative weeks, and instructions about mobility exercises for the shoulders (Fig. 2). The postoperative regimen included no neck collar in both groups. At 6 weeks after surgery, patients returned to the spinal center for a routine visit to the surgeon and at the same time received instructions from the physiotherapist about mobility exercises for the neck (Fig. 2). No more visits to the spinal center were planned after that.

\section{Interventions}

Structured Postoperative Physiotherapy

Six weeks postoperatively, patients randomized to the 


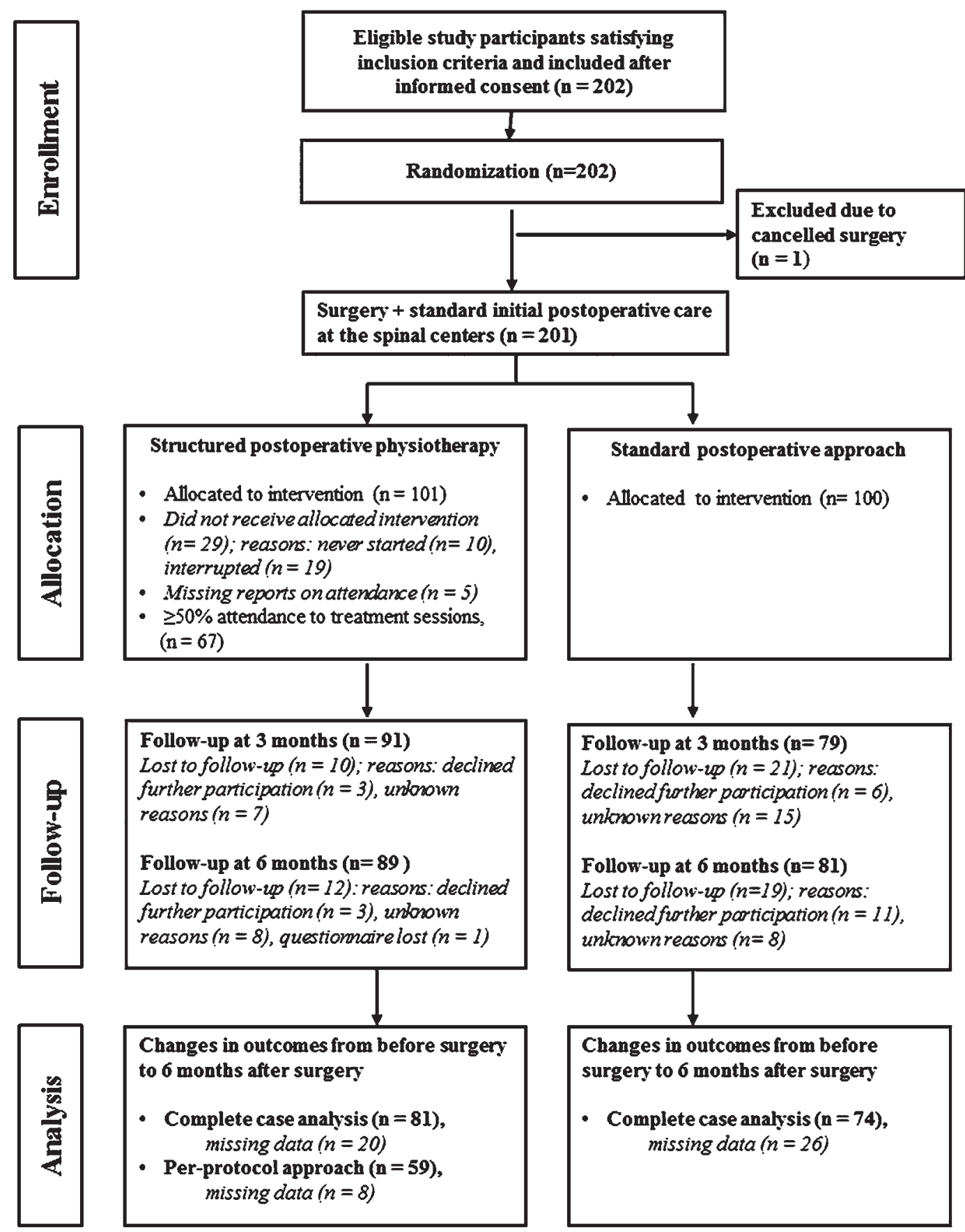

FIG. 1. Patient flow diagram for study inclusion. Analyses were performed in complete-case and per-protocol approaches (comparing patients in the SPT group with at least 50\% attendance at treatment sessions with patients who received the standard postoperative approach).

SPT group were referred to a physiotherapist working in primary care settings in their neighborhood area who was introduced to the study by the project leader through written and oral communication and a half day of practical training. Physiotherapists were recruited on a geographic basis near the patients' home. Structured postoperative physiotherapy included progressive, specific exercises for the neck muscles combined with a cognitive-behavioral approach to address pain and stress management (Fig. 2) and was based on evidence for the management of other neck pain disorders. ${ }^{11,23}$ Within a structured standardized frame, the neck-specific exercises started with nonresistance exercises aimed at activating the deep muscles in the neck. Patients then progressed at individually determined rates to isometric and resistance exercises to improve neuromuscular control and endurance of the neck muscles ${ }^{18}$ (Fig. 2). Patients also performed resistance exercises for the shoulder and trunk muscles (Fig. 2). The cognitivebehavioral approach included a behavioral analysis of patient problems for goal setting and strategies to increase 

movements to avoid, and instructions in mobility exercises for the shoulder; 2) One routine visit to the surgeon and the physiotherapist at 6 weeks postoperative; 3 ) Instructions in mobility exercises for the neck

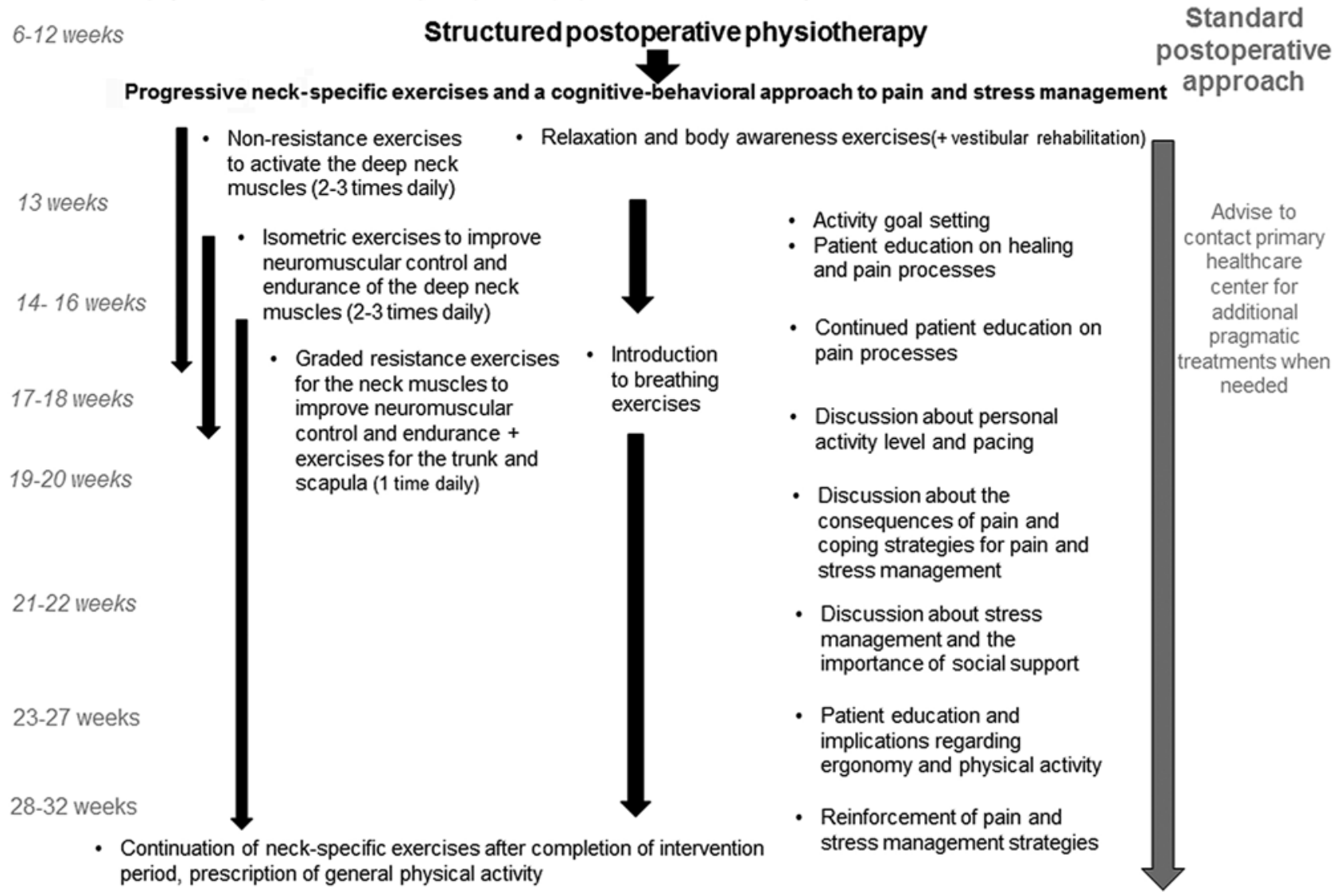

FIG. 2. Summary of standard initial postoperative care at the spinal centers, structured postoperative physiotherapy (SPT), and the standard postoperative approach (SA).

self-efficacy as well as coping strategies (Fig. 2). ${ }^{25}$ The rehabilitation continued for a maximum of 20 weeks, and patients visited the physiotherapist once a week from postoperative Week 6 to Week 12 and twice a week thereafter. Patients were also advised to perform home exercises that were registered in an exercise diary, and the physiotherapists gave feedback on performance during their weekly meetings with the patients. At discharge, patients were encouraged to continue with their home exercises as well as to increase their overall activity level (Fig. 2). Patient adherence rate to treatment sessions was reported by the physiotherapists in the study. Because of the nature of the treatment, neither the patients nor the physiotherapists were blinded.

\section{Standard Postoperative Approach}

The standard postoperative approach from 6 weeks after surgery on was in accordance with Swedish usual postoperative care; patients who felt that they needed additional help were advised to contact their primary health care center for additional treatments, which may have included physiotherapy when needed (Fig. 2). Physiotherapy treatments were pragmatic and not specially designed for the study. The proportion of patients in the SA group who reported additional use of postoperative physiotherapy was recorded.

\section{Data Collection}

Patients completed a preoperative questionnaire that included questions about sociodemographic variables and patient-reported outcome measures, and then they were asked to complete a set of questionnaires at 6 weeks and 3 and 6 months after surgery. This report presents the results of 2 rehabilitation programs. The primary outcome was the Neck Disability Index (NDI) score, and the secondary outcomes were pain intensity and pain frequency in the neck and the arm, global outcome of treatment, expectation fulfillment, and patient enablement. ${ }^{25}$

The results for other collected measures ${ }^{25}$ will be presented later. The questionnaires were sent by postal mail and collected by an independent investigator blinded to the randomization. Reminders were sent to patients who did not return the questionnaire initially and after the patient was considered lost to follow-up. Details about the surgical methods and levels of surgery were collected from medical journals.

\section{Background Variables}

Sociodemographic variables and surgical data for the randomization groups included age, sex, duration of neck and arm pain in months, smoking (yes/no), health-related 
absence resulting from the symptoms (yes/no), use of preoperative neck-related physiotherapy treatments during the last year (yes/no), ACDF versus PCF, and number of operated levels (Table 2).

\section{Outcome Measures \\ Primary Outcome}

The primary outcome of patient-reported neck disability was measured by the NDI. ${ }^{29,31}$ Patient scores on 10 items, including pain intensity, personal care, lifting, sleeping, driving, recreation, headaches, concentration, reading, and work, were summed up to a total score expressed as a percentage, with a higher score indicating a higher degree of disability.

\section{Secondary Outcomes}

Assessment of pain included an evaluation of the intensity of current neck and arm pain using a visual analog scale (VAS) (0-100 mm), where 0 represented "no pain" and 100, "the worst imaginable pain." 5 The frequency of neck and arm pain was assessed on a 5-point scale $(1=$ never, $2=$ sometimes, $3=$ several times per week, $4=$ daily, and $5=$ always), and the scores were dichotomized into either daily symptoms (4-5 points) or occasional symptoms (1-3 points). The global outcome was rated on a 6-point ordinal scale that ranged from "complete recovery" to "much worse," and the answers were dichotomized into substantial improvement (complete recovery or much better) and no or little improvement (better, unchanged, worse, or much worse).$^{16}$ The extent to which participants' expectations for treatment were fulfilled was assessed with the question: "Have your expectations for rehabilitation been fulfilled?" The allowed answers were "yes," "partially," and "no." The Patient Enablement Instrument
$(\mathrm{PEI})^{28}$ was used to evaluate the extent to which participants felt enabled and empowered as a result of receiving treatment. The PEI is a 6-item scale that has been used to evaluate quality of care in general practice. ${ }^{17}$ The highest possible score is 12 , with higher scores indicating greater enablement.

\section{Statistical Analysis}

The required sample size was determined based on preliminary findings for the NDI in a previous study that compared structured physiotherapy alone with surgery combined with structured postoperative physiotherapy for patients with CR. ${ }^{7}$ An expected $10 \%$ between-group difference in the primary outcome of NDI (mean 27, SD 18) with an $\alpha$ level of $5 \%$ and a power goal of $80 \%$ resulted in 60 patients being required for each treatment arm. To allow for dropouts, a total of 202 patients were recruited. ${ }^{25}$ Descriptive statistics are reported as mean and SD, median and interquartile range, or proportions. Preoperative between-group differences were analyzed with parametric or nonparametric statistics depending on the data level. All patients with follow-up data at 6 months were included in a complete case analysis. In a per-protocol approach only patients in the SPT group with a treatment-session attendance rate of more than 50\% (SPT $\geq 50 \%$ ) were included in the analysis. When no more than 1 item was missing in the NDI, the missing value was substituted by the average item score of the questionnaire for that participant; otherwise, the participant was excluded from the analysis. The differences in outcome measures between the treatment groups as determined at the 6-month follow-up assessment were analyzed using the independent t-test, the Mann-Whitney U-test, or the chi-square test, depending on the data level. Differences between the treatment groups

TABLE 2. Background variables for the 201 patients with CR included in the study

\begin{tabular}{|c|c|c|c|c|c|}
\hline \multirow[b]{2}{*}{ Variable } & \multicolumn{2}{|c|}{ SPT Group $(n=101)$} & \multicolumn{2}{|c|}{ SA Group $(n=100)$} & \multirow[b]{2}{*}{$\mathrm{p}$ Value } \\
\hline & No. of Pts & Value & No. of Pts & Value & \\
\hline Age in yrs, mean (SD) & 101 & $50(8.2)$ & 100 & $50(8.7)$ & 0.70 \\
\hline Male sex, $\mathrm{n}(\%)$ & 101 & $51(51)$ & 100 & $54(54)$ & 0.62 \\
\hline Duration of neck pain in mos & 84 & & 89 & & 0.03 \\
\hline Median & & 18 & & 12 & \\
\hline IQR & & $10-36.5$ & & $8-24$ & \\
\hline Duration of arm pain in mos & 84 & & 82 & & 0.02 \\
\hline Median & & 15 & & 12 & \\
\hline IQR & & $10-36$ & & $7-19$ & \\
\hline Smoking, n (\%) & 98 & $24(25)$ & 96 & $25(26)$ & 0.80 \\
\hline Health-related absence from work due to neck/arm symptoms, n (\%) & 92 & $52(57)$ & 86 & $48(56)$ & 0.92 \\
\hline Preop neck-related PT treatments during the last yr, $\mathrm{n}(\%)$ & 95 & $63(66)$ & 92 & $61(66)$ & 0.99 \\
\hline ACDF, $n(\%)$ & 101 & $77(76)$ & 100 & $86(86)$ & 0.08 \\
\hline No. of operated levels, $n(\%)$ & 101 & & 100 & & 0.58 \\
\hline 1 & & $56(55)$ & & $50(50)$ & \\
\hline 2 & & $35(35)$ & & $42(42)$ & \\
\hline$\geq 3$ & & $10(10)$ & & $8(8)$ & \\
\hline
\end{tabular}

$\mathrm{IQR}=$ interquartile range; $\mathrm{SA}=$ standard postoperative approach; SPT = structured postoperative physiotherapy.

Boldface type indicates statistical significance. Group comparisons used independent t-test, Mann-Whitney U-test, or chi-square test depending on the data level. 
in terms of changes in the NDI and changes in VAS scores for neck and arm pain before surgery versus 6 months after surgery were analyzed with repeated-measure analysis of variance (ANOVA) with the Greenhouse-Geisser correction, depending on sphericity. The statistical software package SPSS version 22.0 (IBM Corp.) was used for all analyzes, and the significance level was set at $\mathrm{p} \leq 0.05$.

\section{Results}

\section{Differences Between Treatment Groups at 6 Months' Follow-Up}

At 6 months after surgery, patients in the SPT group reported higher expectation fulfillment $(p=0.01)$ than patients in the SA group. No other differences between treatment groups were found in the complete case analysis ( $p$ $=0.21$ to $\mathrm{p}=0.82$ ). In a per-protocol approach, patients in the SPT group who attended at least $50 \%$ of treatment sessions were found to report less neck pain frequency ( $\mathrm{p}$ $=0.05)$, higher expectation fulfillment $(\mathrm{p}=0.001)$, and greater enablement $(\mathrm{p}=0.04)$ at the 6-month follow-up evaluation than patients in the SA group. There were no other differences between the treatment groups using a per-protocol approach $(\mathrm{p}=0.15$ to $\mathrm{p}=0.58)$. Descriptive statistics for the outcome measures in the treatment groups are presented in Tables 3 and 4.

\section{Between-Group Comparison of Changes in the NDI and VAS Pain Scores}

There was no between-group difference in changes in the NDI scores and VAS scores for neck and arm pain from before surgery to 6 months after surgery in a complete case analysis $(\mathrm{p}>0.43)$ or a per-protocol approach ( $p>0.44)$. The NDI and VAS neck and arm pain scores improved significantly from before surgery to 6 months after surgery in both groups $(\mathrm{p}<0.001)$.

\section{Treatment Groups at Baseline and Patients Lost to Follow-up}

Patients randomized to SPT had a longer preoperative duration of neck and arm pain ( $\mathrm{p}=0.02$ to $\mathrm{p}=0.03)$ and a higher frequency of arm pain symptoms $(\mathrm{p}=0.05)$ compared with patients randomized to SA (Tables 2 and 3). No other differences between the treatment groups were found at baseline $(\mathrm{p}=0.08$ to $\mathrm{p}=0.99)$. No differences were found between the treatment groups at baseline using a per-protocol approach $(\mathrm{p}=0.09$ to $\mathrm{p}=0.82)$. Patients lost to follow-up (15\%) for the primary outcome (i.e., NDI) did not differ in terms of background variables and outcome measures at baseline from patients who were followed up ( $p=0.06$ to $p=0.78)$, except for significantly worse scores on the NDI $(\mathrm{p}=0.004)$ and a lower rate of PCF (7\% vs $21 \%, \mathrm{p}=0.05)$.

\section{Treatment-Session Attendance in the SPT Group}

The physiotherapists in the study reported a treatmentsession attendance rate of $50 \%$ or more in $67 \%$ of the patients who received SPT (SPT $\geq 50 \%, \mathrm{n}=67$, mean age 51 years, SD 8.2 years; 33 men and 34 women). Lack of time and difficulties with absences from work for training $(\mathrm{n}=$

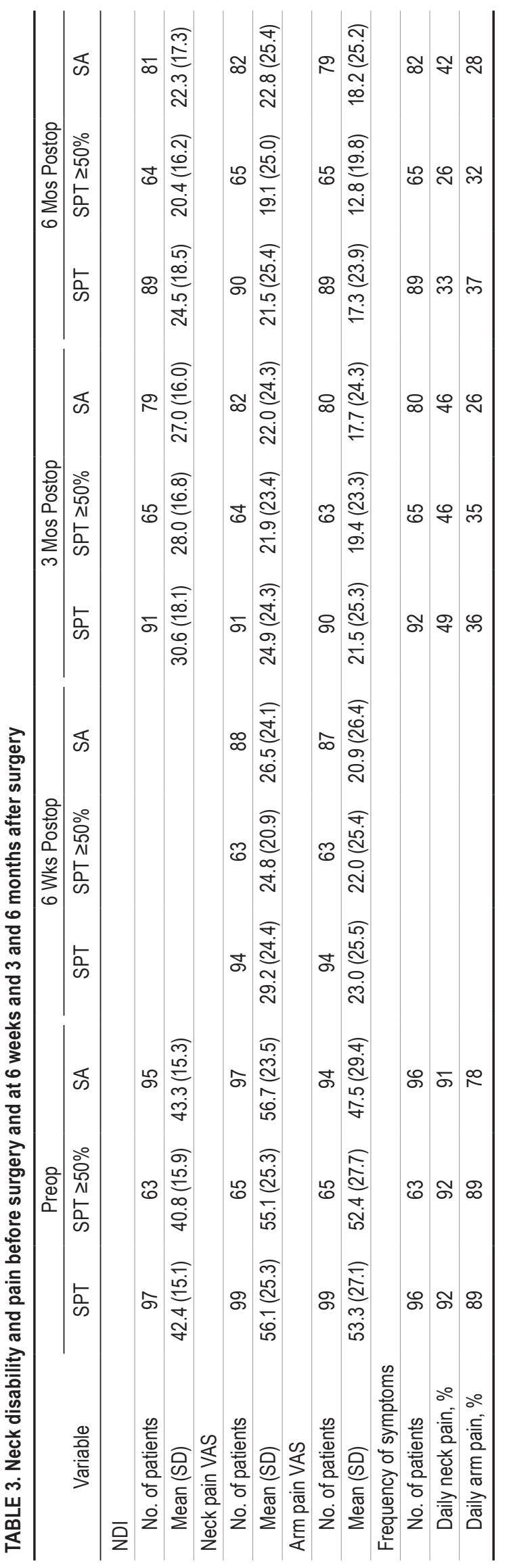


TABLE 4. Global outcome of treatment, expectation fulfillment, and patient enablement at 6 months after surgery

\begin{tabular}{lccc}
\hline \multicolumn{1}{c}{ Variable \& No. of Patients } & SPT & SPT $\geq 50 \%$ & SA \\
\hline Global outcome & & & \\
\hline$\quad$ No. of patients & 89 & 64 & 80 \\
\hline$\quad$ "Substantial improvement," \%* & 60 & 67 & 56 \\
\hline Expectation fulfillment & & & \\
\hline No. of patients & 90 & 65 & 80 \\
\hline Yes, \% & 62 & 71 & 43 \\
\hline Partial, \% & 28 & 23 & 41 \\
\hline No, \% & 10 & 6 & 16 \\
\hline PEI† & & & \\
\hline No. of patients & 79 & 56 & 66 \\
\hline Median & 6.0 & 7.0 & 5.0 \\
\hline IQR & $2-9$ & $4-11$ & $1-8$ \\
\hline
\end{tabular}

* Includes patients who reported being completely recovered or much better.

† Score range $0-12$.

19), not starting the intervention for unknown reasons ( $\mathrm{n}=$ $10)$, and missing reports for attendance rates $(n=5)$ were the reasons for excluding 34 patients (mean age 48 years, SD 8.1 years; 18 men and 16 women) in the per-protocol approach (Fig. 1). No difference was found between the patients excluded in the per-protocol approach and the SPT $\geq$ $50 \%$ subgroup with respect to background variables or outcome measures at baseline $(p=0.11$ to $p=0.71)$, except that there were more smokers among those who were excluded ( $41 \%$ vs $16 \%, p=0.005)$. No serious adverse event during rehabilitation was reported.

\section{Additional Use of Postoperative Physiotherapy in the SA Group}

Additional use of postoperative pragmatic physiotherapy was reported by $61 \%(n=47)$ of the patients who were followed up in the SA group.

\section{Discussion}

This RCT is the first to investigate the additional benefit of structured physiotherapy compared with a standard pragmatic approach in patients who have undergone surgery for cervical disc disease with CR. Except for higher expectation fulfillment in patients who received structured physiotherapy, no between-group differences in outcome measures were found at the 6 months' postoperative followup. In a per-protocol approach, patients in the SPT group who attended at least $50 \%$ of the treatment sessions (SPT $\geq 50 \%$ ) reported less neck pain frequency, higher expectation fulfillment, and greater enablement at the 6-month follow-up assessment compared with patients in the SA group. Moreover, there was a tendency for global outcome and frequency of neck pain to improve more in SPT group during the postoperative period. The importance of supervised treatment sessions for perceived treatment effects has been suggested previously in physiotherapy. ${ }^{12}$ Global outcome has been suggested to reflect patients' judgment of the clinical importance of their changes; ${ }^{9}$ thus this result may reflect changes in domains that are important to the patients following structured physiotherapy that may not have been captured by the other outcome measures. ${ }^{8}$ The long-term effects on future health care utilization of reported greater enablement in patients who received structured physiotherapy should be investigated in future studies.

No differences were found between the treatment groups for patient-reported neck disability and pain intensity in the neck and the arm at the 6-month follow-up using either a complete case analysis or a per-protocol approach. Large improvements in the NDI and VAS neck and arm pain were observed in both groups at 3 months compared with older studies, ${ }^{13,27}$ suggesting improved surgical techniques and patient selection for surgery. Consequently, there may have been limited potential for physiotherapy to further improve NDI and VAS outcomes in the immediate postoperative period. Six months after surgery, $26 \%$ of the patients in the SPT group and $27 \%$ of the patients in the SA group were classified as having no disability (NDI score < $8 \%) .{ }^{20} \mathrm{~A}$ floor effect in the NDI has been suggested to occur when $\geq 15 \%$ of participants achieve the lowest score, ${ }^{21}$ and the responsiveness of the NDI to detect changes in the postoperative period is somewhat controversial. ${ }^{15,30}$

Structured physiotherapy that combines neck-specific exercises with a behavioral approach has been suggested previously as treatment for patients with cervical disc disease and CR preoperatively as well as after surgery during postoperative rehabilitation. ${ }^{7,26}$ The results of this study confirm that patients with CR tolerate neck-specific exercises after surgery and improve over time. In addition, there was a tendency for a slightly better outcome in some variables for patients attending structured physiotherapy sessions compared with those who were offered the option of additional pragmatic therapy as part of a standard approach. The idea that CR patients will tolerate neck-specific training after surgery has been controversial among surgeons and physiotherapists. The results of the present study provide important and new information for the development of a more active and specific rehabilitation approach that hopefully may benefit CR patients in the future. More research within this area is needed. Moreover, more than half of the patients in the SA group reported the additional use of postoperative physiotherapy at 6 months' follow-up. This result suggests that many CR patients perceived a need for additional treatment after surgery, probably because of persistent symptoms, and that more studies are needed to inform evidence-based clinical guidelines for the postoperative treatment of CR patients. However, these patients constitute a heterogeneous group, which includes different subgroups. This may explain why it is difficult to obtain statistically significant and clinically important between-group differences in an RCT of postoperative physiotherapy. More individualized physiotherapy might be needed, and treatment-based approaches may have better effects than a standardized package. In a treatment-based approach those with greater needs after surgery can be identified and offered targeted interventions.

\section{Strengths and Limitations}

This first RCT of postoperative physiotherapy in pa- 
tients with CR had a pragmatic design with the objective of improving external validity as well as generalizability of the results. The involved physiotherapists were recruited on a geographic basis to facilitate patient access to rehabilitation. Larger treatment effects might have been observed with physiotherapists who specialized in musculoskeletal disorders. This would, however, have compromised the generalizability of the results.

Loss of patients to follow-up and missing data are limitations of RCTs. ${ }^{10}$ In the present study, the attrition rate for the primary outcome of NDI at follow-up (15\%) was considered satisfactory. According to standard ethical principles of the Declaration of Helsinki, participation in the study was voluntary, and patients were informed at inclusion that they could discontinue their participation at any time during the study period. Significantly higher scores on the NDI were reported at baseline for the patients lost to follow-up, and future studies should investigate the reasons why some patients decline further participation in rehabilitation studies. The exact effect on the results stemming from this potential bias is uncertain. However, the patients who were lost to follow-up did not differ significantly between randomization groups and they were considered not to have influenced the studied between-group differences. A number of patients in the SPT group either did not start or interrupted the rehabilitation intervention because of problems getting time off from work for rehabilitation once they returned to work. However, 67 patients had a training-session attendance rate of $50 \%$ or more, which could be considered a good rate of attendance and supported the per-protocol analyses.

The sample size estimation used in this study was based on preliminary findings for the NDI scores in a previous study. According to the power analysis, a sample size of 60 participants in each group would be enough to detect a $10 \%$ between-group difference in the primary outcome variable. However, the probability of detecting a $10 \%$ between-group difference in the NDI at 6 months after surgery was questionable, considering that only small changes in the NDI were observed in both groups during the postoperative period. The responsiveness of the NDI to clinically relevant postoperative changes in CR patients may be limited by a potential floor effect in the outcome measure. Thus, the ability for further studies to demonstrate benefits of postoperative physiotherapy may depend on the outcome measure. The preoperative NDI score was comparable to those of other reports, ${ }^{7,27}$ suggesting that the sample was representative of patients with CR undergoing surgery.

The difference between randomization groups in neck and arm symptom duration at baseline occurred by chance, and the influence of symptom duration on postoperative outcomes is not clear. ${ }^{2,24}$ In the present study, symptom duration at baseline was not associated with any outcomes at 6 months ( $p=0.09$ to $p=0.80$ ), except for a significant but very weak association between arm symptom duration at baseline and the NDI score at 6 months (Spearman correlation $\mathrm{r}=0.17, \mathrm{p}=0.05$ ).

Despite its limitations, the present study is unique in that it is the first RCT to investigate postoperative rehabilitation strategies after surgery for CR caused by cervical disc disease. Earlier studies ${ }^{13,14,27}$ reported that up to twothirds of patients have remaining neck-specific disability and low health-related quality of life after surgery for CR. Thus, the present study is of great clinical importance, since it confirms ${ }^{7,26}$ that patients with CR can tolerate the neck-specific exercises that are recommended for other patients with neck pain. ${ }^{11}$ New information-that as many as $61 \%$ of the CR patients in the usual care group sought additional physiotherapy-is also presented.

\section{Conclusions}

In this first RCT of postoperative physiotherapy in patients who have undergone surgery for cervical disc disease with CR, no additional benefit of structured physiotherapy compared with a standard postoperative approach was reported at 6 months after surgery based on patient-reported measures of neck disability, pain, global outcome, or enablement after treatment. Nevertheless, patients who received structured physiotherapy reported greater expectation fulfillment 6 months after surgery compared with patients who received the standard approach, and in a perprotocol analysis, patients in the stuctured physiotherapy group with a training-session attendance rate of $50 \%$ or more reported less frequent neck pain, greater expectation fulfillment, and greater enablement compared with patients in the standard approach group. Many patients in the standard approach group reported additional use of postoperative physiotherapy 6 months after surgery, suggesting that patients who have undergone surgery for cervical disc disease with CR perceive a need for additional postoperative treatment. The results confirm that patients who undergo surgery for cervical disc disease with CR can tolerate neck-specific exercises after surgery, but more studies are needed to inform evidence-based clinical guidelines for the postoperative treatment of these patients.

\section{Acknowledgments}

We acknowledge financial support from the Swedish Research Council, the Swedish Society of Medicine, the Medical Research Council of Southeast Sweden, and the County Council of Region Östergötland, Lions, and $\mathrm{Fu}-$ turum (Academy for Health and Care, Region Jönköping County).

\section{References}

1. Bono CM, Ghiselli G, Gilbert TJ, Kreiner DS, Reitman C, Summers JT, et al: An evidence-based clinical guideline for the diagnosis and treatment of cervical radiculopathy from degenerative disorders. Spine J 11:64-72, 2011

2. Burneikiene S, Nelson EL, Mason A, Rajpal S, Villavicencio AT: The duration of symptoms and clinical outcomes in patients undergoing anterior cervical discectomy and fusion for degenerative disc disease and radiculopathy. Spine J 15:427-432, 2015

3. Carette S, Fehlings MG: Clinical practice. Cervical radiculopathy. N Engl J Med 353:392-399, 2005

4. Caridi JM, Pumberger M, Hughes AP: Cervical radiculopathy: a review. HSS J 7:265-272, 2011

5. Carlsson AM: Assessment of chronic pain. I. Aspects of the reliability and validity of the visual analogue scale. Pain 16:87-101, 1983 
6. Daffner SD, Hilibrand AS, Hanscom BS, Brislin BT, Vaccaro AR, Albert TJ: Impact of neck and arm pain on overall health status. Spine (Phila Pa 1976) 28:2030-2035, 2003

7. Engquist M, Löfgren H, Öberg B, Holtz A, Peolsson A, Söderlund A, et al: Surgery versus nonsurgical treatment of cervical radiculopathy: a prospective, randomized study comparing surgery plus physiotherapy with physiotherapy alone with a 2-year follow-up. Spine (Phila Pa 1976) 38:17151722,2013

8. Evans R, Bronfort G, Maiers M, Schulz C, Hartvigsen J: "I know it's changed": a mixed-methods study of the meaning of global perceived effect in chronic neck pain patients. Eur Spine J 23:888-897, 2014

9. Farrar JT, Young JP Jr, LaMoreaux L, Werth JL, Poole RM: Clinical importance of changes in chronic pain intensity measured on an 11-point numerical pain rating scale. Pain 94:149-158, 2001

10. Fielding S, Fayers P, Ramsay CR: Analysing randomised controlled trials with missing data: choice of approach affects conclusions. Contemp Clin Trials 33:461-469, 2012

11. Gross A, Kay TM, Paquin JP, Blanchette S, Lalonde P, Christie T, et al: Exercises for mechanical neck disorders. Cochrane Database Syst Rev 1:CD004250, 2015

12. Hayden JA, van Tulder MW, Tomlinson G: Systematic review: strategies for using exercise therapy to improve outcomes in chronic low back pain. Ann Intern Med 142:776785,2005

13. Hermansen A, Hedlund R, Vavruch L, Peolsson A: A comparison between the carbon fiber cage and the Cloward procedure in cervical spine surgery: a ten- to thirteen-year follow-up of a prospective randomized study. Spine (Phila Pa 1976) 36:919-925, 2011

14. Hermansen AM, Cleland JA, Kammerlind AS, Peolsson AL: Evaluation of physical function in individuals 11 to 14 years after anterior cervical decompression and fusion surgery-a comparison between patients and healthy reference samples and between 2 surgical techniques. J Manipulative Physiol Ther 37:87-96, 2014

15. Holly LT, Matz PG, Anderson PA, Groff MW, Heary RF, Kaiser MG, et al: Functional outcomes assessment for cervical degenerative disease. J Neurosurg Spine 11:238-244, 2009

16. Hoving JL, Koes BW, de Vet HC, van der Windt DA, Assendelft WJ, van Mameren H, et al: Manual therapy, physical therapy, or continued care by a general practitioner for patients with neck pain. A randomized, controlled trial. Ann Intern Med 136:713-722, 2002

17. Howie JG, Heaney DJ, Maxwell M, Walker JJ: A comparison of a Patient Enablement Instrument (PEI) against two established satisfaction scales as an outcome measure of primary care consultations. Fam Pract 15:165-171, 1998

18. Landén Ludvigsson M, Peolsson A, Peterson G: Neck-Specific Exercise Program. (www.diva-portal.org/smash/get/ diva2:785214/FULLTEXT02.pdf) [Accessed June 6, 2017]

19. Löfgren H, Johansen F, Skogar O, Levander B: Reduced pain after surgery for cervical disc protrusion/stenosis: a 2 year clinical follow-up. Disabil Rehabil 25:1033-1043, 2003

20. MacDermid JC, Walton DM, Avery S, Blanchard A, Etruw E, McAlpine C, et al: Measurement properties of the neck disability index: a systematic review. J Orthop Sports Phys Ther 39:400-417, 2009

21. McCormick JD, Werner BC, Shimer AL: Patient-reported outcome measures in spine surgery. J Am Acad Orthop Surg 21:99-107, 2013

22. Moher D, Hopewell S, Schulz KF, Montori V, Gøtzsche PC, Devereaux PJ, et al: CONSORT 2010 explanation and elabo- ration: updated guidelines for reporting parallel group randomised trials. Int J Surg 10:28-55, 2012

23. Monticone M, Ambrosini E, Cedraschi C, Rocca B, Fiorentini R, Restelli M, et al: Cognitive-behavioral treatment for subacute and chronic neck pain: a Cochrane review. Spine (Phila Pa 1976) 40:1495-1504, 2015

24. Nikolaidis I, Fouyas IP, Sandercock PAG, Statham PF: Surgery for cervical radiculopathy or myelopathy. Cochrane Database Syst Rev 2010:CD001466, 2010

25. Peolsson A, Öberg B, Wibault J, Dedering Å, Zsigmond P, Bernfort L, et al: Outcome of physiotherapy after surgery for cervical disc disease: a prospective randomised multi-centre trial. BMC Musculoskelet Disord 15:34, 2014

26. Peolsson A, Söderlund A, Engquist M, Lind B, Löfgren H, Vavruch L, et al: Physical function outcome in cervical radiculopathy patients after physiotherapy alone compared with anterior surgery followed by physiotherapy: a prospective randomized study with a 2-year follow-up. Spine (Phila Pa 1976) 38:300-307, 2013

27. Peolsson A, Vavruch L, Öberg B: Disability after anterior decompression and fusion for cervical disc disease. Adv Physiother 4:111-124, 2002

28. Rööst M, Zielinski A, Petersson C, Strandberg EL: Reliability and applicability of the Patient Enablement Instrument (PEI) in a Swedish general practice setting. BMC Fam Pract 16:31, 2015

29. Vernon H: The Neck Disability Index: state-of-the-art, 19912008. J Manipulative Physiol Ther 31:491-502, 2008

30. Wibault J, Öberg B, Dedering Å, Löfgren H, Zsigmond P, Persson L, et al: Individual factors associated with neck disability in patients with cervical radiculopathy scheduled for surgery: a study on physical impairments, psychosocial factors, and life style habits. Eur Spine J 23:599-605, 2014

31. Young IA, Cleland JA, Michener LA, Brown C: Reliability, construct validity, and responsiveness of the neck disability index, patient-specific functional scale, and numeric pain rating scale in patients with cervical radiculopathy. Am J Phys Med Rehabil 89:831-839, 2010

32. Zoëga B, Kärrholm J, Lind B: Outcome scores in degenerative cervical disc surgery. Eur Spine J 9:137-143, 2000

\section{Disclosures}

The authors report no conflict of interest concerning the materials or methods used in this study or the findings specified in this paper.

\section{Author Contributions}

Conception and design: Öberg, Löfgren, Zsigmond. Acquisition of data: Wibault, Dedering, Löfgren, Zsigmond, Peolsson. Analysis and interpretation of data: Wibault, Peolsson. Drafting the article: Wibault, Peolsson. Critically revising the article: all authors. Reviewed submitted version of manuscript: all authors. Statistical analysis: Wibault, Peolsson. Funding: Wibault, Peolsson.

\section{Supplemental Information}

\section{Previous Presentations}

Portions of this work were presented in abstract form at the World Confederation for Physical Therapy (WCPT) in Singapore, May $1-4,2015$.

\section{Correspondence}

Johanna Wibault, Department of Medical and Health Sciences, Division of Physiotherapy, Linköping University, Linköping SE-58183, Sweden. email: johanna.wibault@liu.se. 